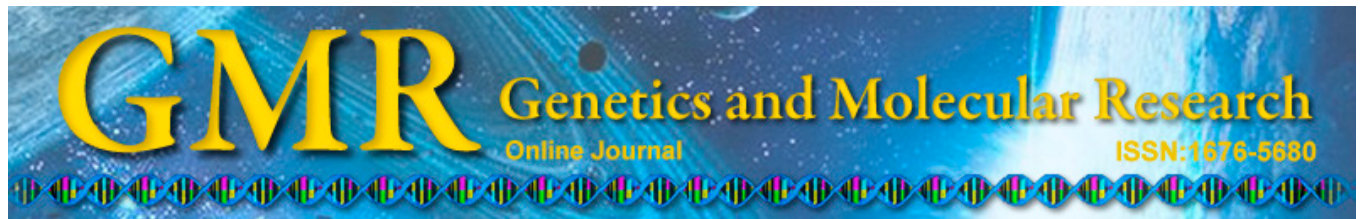

\title{
Selection of an effective microsatellite marker system for genetic control and analysis of gerbil populations in China
}

\author{
X.Y. Du ${ }^{1}$, W. Li ${ }^{1}$, X.Y. Sa ${ }^{2}$, C.L. Li ${ }^{1}$, J. Lu' ${ }^{1}$, Y.Z. Wang ${ }^{3}$ and Z.W. Chen ${ }^{1}$ \\ ${ }^{1}$ Department of Laboratory Animal Science, School of Basic Medical Science, \\ Capital Medical University, Beijing, China \\ ${ }^{2}$ Zhejiang Academy of Medical Science \& Zhejiang Center of Laboratory \\ Animals, Hangzhou, China \\ ${ }^{3}$ Institute of Experimental Animal of Nangfang Hospital, \\ Southern Medical University, Guangzhou, China \\ Corresponding author: Z.W. Chen \\ E-mail: czwen@ccmu.edu.cn \\ Genet. Mol. Res. 14 (3): 11030-11042 (2015) \\ Received February 9, 2015 \\ Accepted May 18, 2015 \\ Published September 21, 2015 \\ DOI http://dx.doi.org/10.4238/2015.September.21.16
}

\begin{abstract}
Although gerbils have been widely used in many areas of biological research over many years, there is currently no effective genetic quality control system available. In the present study, we sought to establish a microsatellite marker system for quality control and conducted an optimized analysis of 137 microsatellite loci in two laboratory gerbil populations and one wild population. Independent sample $t$-tests on the mean effective allele number, mean of Shannon's information index, and mean $H_{\mathrm{E}}$ suggested that 28 of the 137 microsatellite markers were informative for gerbil genetic control. Analysis of 4 laboratory gerbil populations and 1 wild population using the 28 microsatellite loci indicated that allele numbers varied from 1.9639 (Guangzhou, GZ) to 6.6071 (North-West wild, NW). The average of $H_{\mathrm{O}}$ versus $H_{\mathrm{E}}$ was $0.6236 / 0.3802,0.6671 / 0.4159,0.4185 / 0.3464,0.4592 / 0.3821$, and 0.3972/0.4167 for the Beijing, NW, Hangzhou, Dalian, and GZ popula-
\end{abstract}


tions, respectively. The GZ population showed the greatest differentiation, having higher $R_{\mathrm{ST}}$ and Nei's standard genetic distances. An AMOVA revealed high genetic differentiation among the five populations $\left(F_{\mathrm{ST}}=0.296\right)$. The microsatellite system established here is effective and will be important in future studies for genetic quality control and monitoring of gerbil breeds.

Key words: Microsatellites; Population variation; Genetic control; Genetic differentiation; Gerbil

\section{INTRODUCTION}

The Mongolian gerbil (Meriones unguiculatus) is an important laboratory animal that has been widely used as an animal model for human diseases (Levine and Sohn, 1969; Hossmann, 1998). The species was first introduced into the laboratory in 1935 when 20 pairs were captured in eastern Mongolia and imported to Japan. Some descendant pairs were exported to the United States in 1954 and formed the breeding stock for laboratories there. In China, there are 4 stocks of laboratory gerbils separately located at the Zhejiang Laboratory Animal Centre (Ding et al., 2008), Dalian Medical University, Guangzhou Medical University, and Capital Medical University. Currently, the gerbil is proving particularly useful for studying strokes because of the lack of communicating arteries between the posterior vertebral arteries (through the basilar artery) and the 2 internal carotid arteries anteriorly. Without significant collateral flow from the vertebral blood supply to the forebrain, a transient bilateral common carotid artery occlusion induces consistent ischemic injury (Kirino and Sano, 1984; Du et al., 2011). The relative simplicity of carotid occlusion makes this model useful for the study of brain ischemic mechanisms (Dowden and Corbett, 1999; Beck et al., 2007). Since 1996, Mongolian gerbils infected with Helicobacter pylori (Hirayama et al., 1996) have proven very useful for mimicking human infections, for example, for analyzing underlying processes and studying gastritis and gastric cancer (Shibata et al., 2006; Chiba et al., 2008). The gerbil has also been used to study nutrition (Deming et al., 2000), aging (Cheal, 1986), epilepsy (Fujisawa et al., 2003), parasitic infection (Kato et al., 2005; Conchedda et al., 2006), viral infections (Song, 1999; Watanabe et al., 2001). Recently, the gerbil has been used for studies of neoplastic prostate lesions as its prostate has compact lobes, somewhat similar to the human prostate, and unlike that of rats and mice which have distinct lobes (Cordeiro et al., 2008; Pereira et al., 2009). However, genetic studies in the gerbil are hampered by the lack of a reliable genetic marker system. Genetic variations among different strains can limit the value of gerbil models by increasing the heterogeneity of their experimental responses. Hence, the establishment of a genetic monitoring system for gerbils is important.

Microsatellites, also known as simple sequence repeats (SSRs), are polymorphic loci consisting of short tandem repeated motifs of 1 to 6 bases randomly distributed throughout mammalian genomes. Microsatellites are highly polymorphic and are more random in their distribution throughout the genome than variable nucleotide tandem repeats (VNTRs) and restriction fragment length polymorphism (RFLPs) (Goodfellow, 1993). Microsatellites are widespread in eukaryotic genomes and are currently being used as genetic markers in cows (Beckmann and Soller, 1990), horses (Marklund et al., 1994), humans (Weber, 1990), and other species. Furthermore, microsatellites have been successfully used for linkage studies 
in both humans and mice, and have enabled the identification of numerous quantitative trait loci (QTLs) associated with polygenic disorders (Vyse and Todd, 1996; Mogil, 1999). The abundance of the repeats and their variability make them very valuable for genetic monitoring. Primer sets for the flanking regions of microsatellite repeat sequences have been developed for the mouse and rat, enabling easy implication of markers by PCR (Love et al., 1990; Dietrich et al., 1996).

A few studies have examined genetic diversity in gerbils. However, these studies were based on a limited number of populations, or used a limited number of genetic markers, or both (Neumann et al., 2001). More recently, the CMU, HOH, and YIN populations were investigated but details of the markers were not provided, such as the number of alleles and the sizes of allele fragments (Du et al., 2010). The failure to establish a suitable genetic quality control method for gerbils prompted the present study. We sought to develop a method for analyzing the genetic characteristics of gerbil populations in China. To this end, we screened a number of microsatellite loci and selected 28 informative markers. We then tested the efficiency of these markers in gerbils from four laboratory populations and one wild population. Here, we present a description of the genetic characteristics of these gerbils as determined by the selected microsatellite markers.

\section{MATERIAL AND METHODS}

\section{Sample collection}

We collected liver or kidney samples from 164 gerbils (133 animals from 4 laboratory animal centers and 31 from a wild gerbil population) in China. The laboratory populations came from Beijing ( $\mathrm{BJ}, \mathrm{n}=31)$, Hangzhou $(\mathrm{HZ}, \mathrm{n}=12+30,12$ for selecting microsatellite loci and 30 for population analysis), Dalian (DL, $n=30)$, and Guangzhou $(\mathrm{GZ}, \mathrm{n}=30)$. The wild population came from the North-West $(\mathrm{NW}, \mathrm{n}=31)$. The locations of the sampled gerbil populations are shown in Figure 1. Samples were stored at $-80^{\circ} \mathrm{C}$. The BJ samples were from the breeding colony of the Department of Laboratory Animal Science, Capital Medical University, Beijing, China. The HZ samples were from the Zhejiang Center of Laboratory Animals. The DL population was acquired from Dalian Medical University. The GZ samples came from Guangzhou Medical University. The 31 wild gerbils were recent captures from the junction of the Inner Mongolia Autonomous Region and Ningxia Province within a range of $400 \mathrm{~km}$.

Microsatellite markers for genetic monitoring were identified in a two-step selection process. First, we used genomic DNA from the $12 \mathrm{HZ}$ samples to amplify 137 microsatellite markers previously described in gerbils (Neumann et al., 2001; Du et al., 2010). Thirty-nine of these markers showing higher allele numbers and greater polymorphism were selected in agarose gel electrophoresis assays. Second, microsatellite genotyping of the BJ, NW, and HZ populations was performed using the 39 microsatellite markers by short tandem repeat (STR) scanning. We calculated the mean effective allele number, the mean of Shannon's information index and the mean of $H_{\mathrm{E}}$ for different numbers of microsatellite markers in the $\mathrm{BJ}, \mathrm{NW}$, and $\mathrm{HZ}$ populations in order to determine the optimal microsatellite marker system that uses the lowest number of markers but enables reliable genetic quality monitoring of the gerbils. The optimized system contained 28 markers and was used for microsatellite genotyping of DL and GZ. 


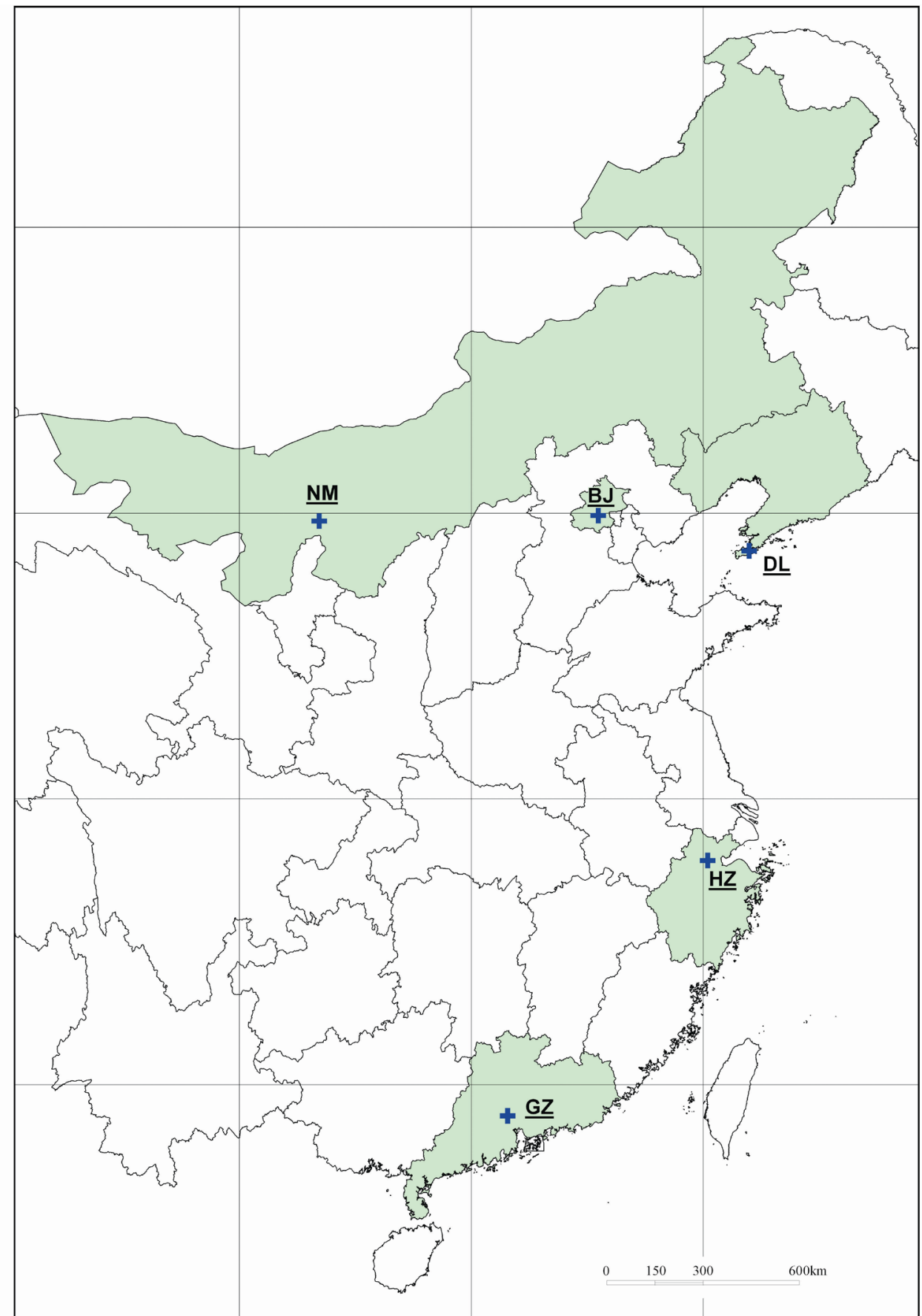

Figure 1. Locations of the gerbil populations used in the present study. The four laboratory populations are: BJ, Beijing; HZ, Hangzhou; DL, Dalian; and GZ, Guangzhou. The wild gerbil population (NW) originated from the North-West of China. 


\section{DNA extraction, PCR, and microsatellite genotyping}

Genomic DNAs were extracted from the frozen liver or kidney samples of the 164 gerbils following the method described in (Du et al., 2010). In brief, $100 \mathrm{mg}$ tissue was cut into pieces and placed in $1 \mathrm{~mL}$ STE buffer $(10 \mathrm{mM}$ Tris-HCl, $\mathrm{pH}$ 8.0; $50 \mathrm{mM}$ EDTA, pH 8.0; $200 \mathrm{mM} \mathrm{NaCl} ; 0.5 \%$ SDS) and digested with $0.02 \mathrm{mg} / \mathrm{mL}$ proteinase $\mathrm{K}$ at $55^{\circ} \mathrm{C}$ for $12 \mathrm{~h}$. The digestion mixture was then extracted with phenol:chloroform (ratio $=1: 1$ ). The DNA was precipitated with ethanol and dissolved in TE buffer. The resulting DNA was quantitated with a Microplate Absorbance Reader (Bio-Rad 680, USA), evaluated by agarose gel electrophoresis, and stored at $-20^{\circ} \mathrm{C}$.

As described above, 39 of 137 microsatellite markers showed higher allele numbers and more polymorphism. These were used for microsatellite genotyping of BJ, NW, and HZ populations. The forward primers for these markers were labeled at the $5^{\prime}$ end with FAM, TAMRA, or HEX fluorescent dyes. PCR was performed using a total volume of $20 \mu \mathrm{L}$ that contained $50 \mathrm{ng}$ genomic DNA, $0.5 \mu \mathrm{M}$ fluorescently labeled forward primer and $0.6 \mu \mathrm{M}$ reverse primer, $2 \mu \mathrm{L}$ Applied Biosystems 10X buffer B with $\mathrm{MgCl}, 200 \mu \mathrm{M}$ deoxynucleoside triphosphates, and 1.0 U Taq DNA polymerase. The amplification conditions were initial denaturation at $94^{\circ} \mathrm{C}$ for $5 \mathrm{~min}$, followed by 35 cycles of denaturation at $94^{\circ} \mathrm{C}$ for $30 \mathrm{~s}$, annealing at a primer-specific gradient temperature for $30 \mathrm{~s}$, extension at $72^{\circ} \mathrm{C}$ for $30 \mathrm{~s}$, with a final extension at $72^{\circ} \mathrm{C}$ for $10 \mathrm{~min}$. The PCR products were first assessed on $1.5 \%$ agarose gels stained with $10 \mu \mathrm{g} / \mu \mathrm{L}$ ethidium bromide and visualized using a UV transilluminator (Vilber Lourmat Inc.). Genotyping was conducted on an ABI-3730XL DNA Analyzer (PE Biosystems, USA). We used GENEMARKER version 1.5 with a 500-bp size standard to determine allele sizes.

\section{Statistical analysis}

We used Popgene 3.2 (Raymond and Rousset, 1995) to test deviations from the HardyWeinberg equilibrium (HWE) for each locus over all populations and for all loci using an exact test based on a Markov chain algorithm with a forecasted chain length of 10,000 dememorizations, 100 batches, and 5000 iterations per batch. Significance levels were calculated per locus, per population, and over all loci and populations combined. Genetic diversity within populations was measured as the mean number of alleles $\left(N_{\mathrm{A}}\right)$ per locus. $H_{\mathrm{O}}$ and $H_{\mathrm{E}}$ were calculated and Wright's (Nei 1987) fixation index (Fis) as a measure of heterozygote deficiency or excess was tested based on allele frequencies (10,000 dememorizations, 100 batches, and 5000 iterations per batch) using the Popgene 3.2 software. The genetic distances between the five populations were measured according to the method of Nei's standard distance (Nei 1972). A dendrogram using UPGMA for neighbor joining, based on Nei's unbiased genetic distance, was generated in the Popgene 3.2 software. The tree was visualized using TREEVIEW (Page 1996).

We used SPSS Statistics 17.0 to perform independent sample $t$-tests on the mean of effective allele number, the mean of Shannon's information index, and the mean of $H_{\mathrm{E}}$ of different numbers of microsatellite markers in BJ, NW, and HZ samples, to determine the optimum microsatellite marker system with the fewest markers for the genetic quality monitoring of closed gerbil colonies.

Genotypic linkage disequilibrium was estimated between all locus pairs with ARLEQUIN 3.0 (Excoffier et al., 2005) (Markov chain using 5000 dememorizations, 100 batches, and 500 iterations per batch). The pairwise $R_{\mathrm{ST}}$ was estimated by ARLEQUIN 3.0 (Paetkau 
et al., 1995, 1997; Waser and Strobeck 1998). To estimate the proportion of genetic variance explained by the populations, we also conducted an analysis of molecular variance (AMOVA; 10,000 permutations) by ARLEQUIN 3.0. The AMOVA examined the hierarchical proportion of total genetic variance in "among populations within groups", "among individuals within populations", and "within individuals".

\section{RESULTS}

\section{Optimum microsatellite marker system}

Thirty-nine of the 137 markers analyzed had more than 3 alleles (Table 1). The independent sample $t$-tests on mean effective allele number, mean of Shannon's information index, and mean of $H_{\mathrm{E}}$, showed that there were no significant differences in the BJ, NW, or HZ populations (Tables $\mathbf{S 1}, \underline{\mathbf{S} 2}$, and $\underline{\mathbf{S 3}})$ based on either 28 or 39 microsatellite markers $(\mathrm{P}>$ 0.05 ). The independent sample $t$-tests of the three indicators based on 27 microsatellite markers in CMU and HZ populations also showed no significant differences from that based on 39 microsatellite markers. However, the independent sample $t$-tests on mean of $H_{\mathrm{E}}$ in the NW population based on 27 microsatellite markers was significantly different from that based on 39 microsatellite markers $(\mathrm{P}>0.05)$; the other indicators did not show a significant difference.

\section{Genetic diversity and tests of disequilibrium}

The NW population had the largest total number of alleles (185), while the HZ population had the fewest (100). The overall number of alleles per locus ranged from 9 (GU562695 and GU562777) to 46 (AF200946). $H_{\mathrm{E}}$ was the highest in the NW population $(0.6671)$ and the lowest in GZ (0.3972) (Table 2), while the $H_{\mathrm{O}}$ was the highest in the GZ population $(0.4167)$ and the lowest in $\mathrm{HZ}(0.3464) . H_{\mathrm{O}}$ values were lower than $H_{\mathrm{E}}$ in most populations; only the GZ population had an $H_{\mathrm{O}}$ value higher than $H_{\mathrm{E}}$ (Table 2). These results indicate that either random mating has not occurred in the GZ population or that there are additional subdivisions that are unknown or unidentified. The ARLEQUIN analysis did not find linkage disequilibrium in any of the 5 populations.

The average $H_{\mathrm{O}}$ was less than the expected value (0.3883 and 0.5132 , respectively). Significant high heterozygosity was found at most loci in all populations (Table 2). A probability test using the Markov chain method, based on the 'exact HW test' for each locus over all sampling sites, identified obvious departures from the HWE at all 28 loci after application of the Bonferroni correction. All populations displayed significant departures from the HWE $(\mathrm{P}<$ 0.01 ); the deviations suggested that the laboratory animal populations were insufficiently large or that random mating did not occur. There was no clear evidence for linkage disequilibrium between any two of the 28 loci after correcting for multiple testing.

Pairwise $R_{\mathrm{ST}}$ values between GZ and other populations (0.3345-0.5197) were higher than in most pairs of populations $(0.1772-0.4228$ with $\mathrm{P}<0.01)$, except for GZ and DL populations (0.3690) (Table 3$)$. The $D_{\mathrm{S}}$ values between the GZ and other populations (0.6414$1.10184)$ were higher than in most other combinations of populations $(0.7286-0.9681)$, except for GZ and BJ populations (0.6414) (Table 3). The phylogenetic tree constructed using $D_{\mathrm{S}}$ values (Figure 2) showed that the GZ and BJ populations were genetically the most differentiated of the sampled populations. 
Table 1. Characteristics of 39 selected gerbil loci.

\begin{tabular}{|c|c|c|c|c|c|}
\hline $\mathrm{AN}$ & Primer sequences $\left(5^{\prime}-3^{\prime}\right)$ & $\mathrm{Mg}^{2+}$ & $\mathrm{AT}\left({ }^{\circ} \mathrm{C}\right)$ & $N_{\mathrm{A}}$ & Chr. \\
\hline \multirow[t]{2}{*}{ AF200942 } & CAGGCACCCCCAGTTT & 2.0 & 54 & $29^{\mathrm{a}}$ & / \\
\hline & GTCTACACAGGCTGAGGATGT & & & & \\
\hline \multirow[t]{2}{*}{ AF200943 } & GGCTCCTGATTCTACATTTCT & 2.0 & 57 & $30^{\mathrm{a}}$ & I \\
\hline & CAACCATTGGCAACTCTC & & & & \\
\hline \multirow[t]{2}{*}{ AF200944 } & GCTGGGCTTTAATGTTTATTT & 2.0 & 54 & $28^{\mathrm{a}}$ & / \\
\hline & GGTGGCTCACACTTTCTGT & & & & \\
\hline \multirow[t]{2}{*}{ AF200946 } & TTTCTGGGGTCTCTTTCTCTC & 2.0 & 57 & $46^{\mathrm{a}}$ & / \\
\hline & CCATTCTGCAAGACTCCTCT & & & & \\
\hline \multirow[t]{2}{*}{ AF200945 } & AGTCCCTATTACATCCACAAG & 2.0 & 57 & $29^{\mathrm{a}}$ & / \\
\hline & TTATCCTGCAAAGCCTAAG & & & & \\
\hline \multirow{2}{*}{ AF200941 } & TGGGTCCTTTGGAAGA & 2.0 & 55 & $33^{\mathrm{a}}$ & / \\
\hline & TGGCTTAAAATGAATCACTTA & & & & \\
\hline \multirow{2}{*}{ AF200947 } & GACAGAGTGGGAGGGGTATGT & 2.0 & 55 & $26^{\mathrm{a}}$ & / \\
\hline & TGGCAAGTTTGGTTTGTTTGA & & & & \\
\hline \multirow{2}{*}{ GU562707 } & CTGCCACCCCTGAACCATTA & 2.0 & 52.6 & $16^{\mathrm{a}}$ & D16Mit7 \\
\hline & CTACAAGATGTGGGGCATGA & & & & \\
\hline \multirow[t]{2}{*}{ GU562706 } & CAGGAATAAAGTATAATGGGGTGC & 2.0 & 49.1 & $12^{\mathrm{a}}$ & D16Mit26 \\
\hline & CCCATGATCAGTTGGGTTTT & & & & \\
\hline \multirow[t]{2}{*}{ GU562805 } & TGTGTGACTGCTTGGAAGATG & 1.5 & 50.0 & $15^{\mathrm{a}}$ & D1Mit362 \\
\hline & CTGAGTCCCTAAAGTTGTCCTTG & & & & \\
\hline \multirow[t]{2}{*}{ GU562724 } & GTTTTTCTCAGAAGAATGCAATATACC & 2.0 & 48.1 & $14^{\mathrm{a}}$ & D8Mit184 \\
\hline & TGAGAAGAATGAGGAATTTGTCC & & & & \\
\hline \multirow[t]{2}{*}{ GU562729 } & TCTGAAGTTTGAATGGTTGTGG & 2.0 & 47.3 & $17^{\mathrm{a}}$ & D7Mit33 \\
\hline & TTTCAAAATCGTGTCATTTTGC & & & & \\
\hline \multirow[t]{2}{*}{ GU562736 } & AAAGAATTGCACATCCACTGG & 2.0 & 47.0 & $26^{\mathrm{a}}$ & D6Mit37 \\
\hline & TGCCCAGGATGTTTAAGAGG & & & & \\
\hline \multirow[t]{2}{*}{ GU562772 } & TCAGGGCTCTCTAAGGGACA & 2.0 & 53.1 & $12^{\mathrm{a}}$ & D5Mit31 \\
\hline & ACTATGCAGCCACCAAATCC & & & & \\
\hline \multirow[t]{2}{*}{ GU562774 } & CCACTGGATGGCAACAGAC & 2.0 & 53.1 & $18^{\mathrm{a}}$ & D12Mit201 \\
\hline & TATGTGTTTCAAAACCACACTCG & & & & \\
\hline GU562777 & GCTCCСТTTCСТCTTGAACC & 2.0 & 49.1 & $9^{\text {a }}$ & D2Mit22 \\
\hline & GGGCCCTTATTCTATCTCCC & & & & \\
\hline GU562790 & AGGAGAGAACCAACTGCTGC & 2.5 & 59.8 & $26^{\mathrm{a}}$ & D15Mit124 \\
\hline & GGCCAGTGATGACTTTATAATGC & & & & \\
\hline GU562714 & CCAGAACTTTTGCTGCTTCC & 2.0 & 58.7 & $17^{\mathrm{a}}$ & D11Mit36 \\
\hline & GTGAGCCCTAGGTCCAGTGA & & & & \\
\hline GU562731 & CCACCTGGAATACATGTAACCC & 2.0 & 49.1 & $12^{\mathrm{a}}$ & D7Mit71 \\
\hline & TAAGATCCAAGAGATGGGTTAAGC & & & & \\
\hline GU562751 & CTCAAGTCTCACTTCTCTGCACA & 2.0 & 47.3 & $26^{\mathrm{a}}$ & D4Mit5 \\
\hline & ACACCCAAGGTTGACCTCTG & & & & \\
\hline GU562747 & AACACATGAAACGTGTGCGT & 2.0 & 50.6 & $13^{\mathrm{a}}$ & D3Mit130 \\
\hline & TGATAGGCATGCTTAAGCCC & & & & \\
\hline GU562700 & AATCCTTGTTCACTCTATCAAGGC & 2.0 & 49.1 & $16^{\mathrm{a}}$ & D19Mit1 \\
\hline & CATGAAGAGTCCAGTAGAAACCTC & & & & \\
\hline GU562713 & AGTAACATGGAACATCGACGG & 2.0 & 48.1 & $26^{\mathrm{a}}$ & D11Mit35 \\
\hline & TGCTCAGCTCTGGAGTGCTA & & & & \\
\hline GU562704 & CCTCTGAGGAGTAACCAAGCC & 2.0 & 52.6 & $14^{\mathrm{a}}$ & D17Mit38 \\
\hline & CACAGAGTTCTACCTCCAACCC & & & & \\
\hline GU562695 & CCTGTTTGGGCACCTAGATT & 2.0 & 48.1 & $9^{\mathrm{a}}$ & DXMit17 \\
\hline & TAATAACCCATGTTTTCTGTGGG & & & & \\
\hline GU562725 & ACACTCAGAGACCATGAGTACACC & 2.0 & 50.6 & $10^{\mathrm{a}}$ & D8Mit56 \\
\hline & GAGTTCACTACCCACAAGTCTCC & & & & \\
\hline GU562718 & TCTCCTTGGAATTCACAGCC & 2.0 & 54.7 & $14^{\mathrm{a}}$ & D10Mit66 \\
\hline & GACATTCCTTAAGAGAGACAGTCC & & & & \\
\hline GU562810 & TCATTCAACATTCTGTCAATCG & 2.0 & 49.0 & $17^{\mathrm{a}}$ & D13Mit1 \\
\hline & CACAACAAGGTTAACCTCTAGACA & & & & \\
\hline GU562750* & CCACGGAGAACAACTGAAGA & 2.0 & 50.6 & $7^{\mathrm{b}}$ & D3Mit17 \\
\hline & CAAGAACCATGGAGCCATG & & & & \\
\hline GU562709* & GCGTCACTGAAAGTAGGGAG & 2.0 & 53.6 & $17^{\mathrm{b}}$ & D15Mit17 \\
\hline & GGTTCAGGATTGGGGTACA & & & & \\
\hline GU562715* & ACTAGCCATATGGTTTCTGATGG & 2.0 & 49.1 & $3^{\mathrm{b}}$ & D11Mit42 \\
\hline & AAAGCTCACAGCCCTGCTAC & & & & \\
\hline
\end{tabular}

Continued on next page 


\section{Table 1. Continued.}

\begin{tabular}{|c|c|c|c|c|c|}
\hline $\mathrm{AN}$ & Primer sequences $\left(5^{\prime}-3^{\prime}\right)$ & $\mathrm{Mg}^{2+}$ & $\mathrm{AT}\left({ }^{\circ} \mathrm{C}\right)$ & $N_{\mathrm{A}}$ & Chr. \\
\hline GU562703* & $\begin{array}{l}\text { GATCCAGACCACACCCCCTCACCA } \\
\text { CСТTCAAGCTTGGCTCTCAAAGGA }\end{array}$ & 2.0 & 58.0 & $8^{\mathrm{b}}$ & D17Mit13 \\
\hline GU562698* & $\begin{array}{l}\text { CCGTCTGTATAGTTCCTTTGCC } \\
\text { AATTCAGGCACTCACAAGGG }\end{array}$ & 2.0 & 50.6 & $3^{\mathrm{b}}$ & D19Mit9 \\
\hline GU562712* & $\begin{array}{l}\text { AAGCCAGCCCAGTCTTCATA } \\
\text { TGCTGTACTGATGACCCACTG }\end{array}$ & 2.0 & 56.6 & $7^{\mathrm{b}}$ & D11Mit4 \\
\hline GU562744* & $\begin{array}{l}\text { CCTAAAAAGTGTCTTCTGACCTCC } \\
\text { TGAGAGGTAGAGACAGAGGCG }\end{array}$ & 2.0 & 49.1 & $12^{\mathrm{b}}$ & D4Mit5 \\
\hline GU562779* & $\begin{array}{l}\text { CTAGCTATGTGATGAGTGTGTCG } \\
\text { TGGATGTTTTTGGAAATCCTGA }\end{array}$ & 2.0 & 53.1 & $9^{b}$ & D3Mit56 \\
\hline GU562815* & $\begin{array}{l}\text { CTGAAAACTTCCAAGTCACTCTG } \\
\text { CAAGCAGAAAAGCAAACCAG }\end{array}$ & 2.0 & 53.1 & $9^{b}$ & D15Mit267 \\
\hline GU562782* & $\begin{array}{l}\text { CTGGGTAGCCAACCATGTT } \\
\text { TGCCATATGCCAAAACAGAC }\end{array}$ & 2.5 & 49.1 & $9^{\mathrm{b}}$ & D6Mit9 \\
\hline GU562798* & $\begin{array}{l}\text { AAGTATGAGCTATGGCACCTAGC } \\
\text { GTTTGTGTGCCTCTGCATGT }\end{array}$ & 2.5 & 58.0 & $6^{\mathrm{b}}$ & D7Mit39 \\
\hline
\end{tabular}

AN: GenBank accession No. Optimized annealing temperature (AT) and concentration of $\mathrm{Mg}^{2+}(\mathrm{mM})$ for PCR; $N_{\mathrm{A}}$ : number of alleles; Chr.:position on mouse chromosome of the corresponding mouse locus; ${ }^{*}$ loci that were removed from the optimized microsatellite marker system; aallele number in 5 populations (BJ, NW, HZ, DL, GZ) at each locus; ballele number in 3 populations (BJ, NW, HZ) at each locus.

Table 2. Sample size, number of alleles $\left(N_{\mathrm{A}}\right)$, Shannon's information index (I), expected and observed heterozygosity $\left(H_{\mathrm{E}}, H_{\mathrm{O}}\right)$, number of HW loci averaged over 28 loci and five gerbil populations.

\begin{tabular}{lcccccc}
\hline Population & Sample size $(\mathrm{N})$ & $N_{\mathrm{A}}$ & $\mathrm{I}$ & $H_{\mathrm{E}}$ & $H_{\mathrm{O}}$ & No of HW Loci \\
\hline BJ & 31 & 4.6071 & 1.1863 & 0.6239 & 0.3802 & 5 \\
NW & 31 & 6.6071 & 1.3964 & 0.6671 & 0.4159 & 4 \\
HZ & 30 & 3.5714 & 0.7491 & 0.4185 & 0.3464 & 6 \\
DL & 30 & 2.3326 & 0.8443 & 0.4592 & 0.3821 & 6 \\
GZ & 30 & 1.9639 & 0.7350 & 0.3972 & 0.4167 & 9 \\
\hline
\end{tabular}

BJ, Beijing; NW, North-West wild; HZ, Hangzhou; DL, Dalian; GZ, Guangzhou.

An AMOVA demonstrated that variance among individuals within populations accounted for $69.82 \%$ of the variation, while variance among populations was estimated as $29.62 \%$ $\left(0.29<F_{s t}>0.17\right.$, Table 4$)$, indicating that all the populations were differentiated from each other.

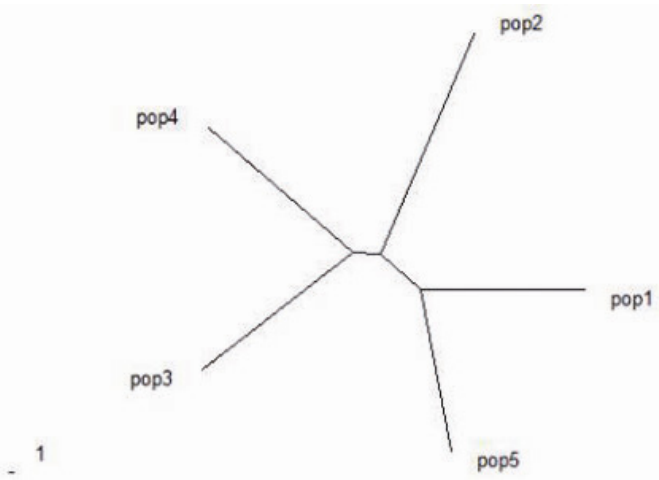

Figure 2. Neighbor-joining tree based on Nei's standard genetic distance (corrected for sample size; Nei et al. 1987) for the 5 gerbil populations studied here. Pop1 is BJ, pop2 is NW, pop3 is HZ, pop4 is DL, and pop5 is GZ. 
Table 3. Pairwise $R_{\mathrm{ST}}$ values (lower matrix) and $D_{\mathrm{S}}$ values (upper matrix) between five gerbil populations.

\begin{tabular}{lcccrr}
\hline Population & BJ & NW & HZ & DL & GZ \\
\hline BJ & & 0.7286 & 0.7473 & 0.7186 & 0.6414 \\
NW & 0.1772 & 0.3280 & 0.9254 & 0.7351 & 1.0184 \\
HZ & 0.2155 & 0.2440 & 0.4228 & 0.9681 \\
DL & 0.2596 & 0.3597 & 0.5197 & 0.3690 & 0.9246 \\
GZ & 0.3345 & & & \\
\hline
\end{tabular}

BJ, Beijing; NW, North-West wild; HZ, Hangzhou; DL, Dalian; GZ, Guangzhou.

Table 4. Analysis of molecular variance (AMOVA) for 28 microsatellite markers in five gerbil populations.

\begin{tabular}{|c|c|c|c|c|c|c|}
\hline Source of variation & df & Sum of squares & Variance & Fixation indices & Variation $(\%)$ & $P$ value \\
\hline Among populations & 4 & 10941098.91 & 41740.62796 & $\mathrm{FST}=0.29616$ & 29.62 & $<0.01$ \\
\hline Among individuals within populations & 147 & 29048568.91 & 98408.70057 & $\mathrm{FIS}=0.99202$ & 69.82 & $<0.01$ \\
\hline Within individuals & 152 & 120370.5 & 791.91118 & $\mathrm{FIT}=0.99438$ & 0.56 & $<0.01$ \\
\hline Total & 303 & 40110038.32 & 140941.2397 & & & \\
\hline
\end{tabular}

\section{DISCUSSION}

The Mongolian gerbil (Meriones unguiculatus) has been widely used as a valuable animal model of several human diseases (Levine and Sohn, 1969). As a consequence of its use as a model system in biomedical research, genetic quality control has become increasingly important for laboratory populations. In the present study, we sought to establish a genetic quality testing system using microsatellite markers for the gerbil populations in China as this would enable genetic quality control and monitoring of these populations. Typically, there are three important considerations for the establishment of a microsatellite genetic monitoring system: high polymorphism, uniform distribution across the chromosomes, and an abundance of markers. It is important that the selected microsatellite markers are highly polymorphic in order to effectively detect genetic diversity. In addition, the selected microsatellite markers need to be uniformly distributed across all chromosomes to genetically characterize the population. At present, the distribution of the microsatellite markers across gerbil chromosomes is still unknown, and so it has to be inferred from the known distributions in mice. Use of large numbers of microsatellite markers produces more accurate results but is time-consuming and expensive. In contrast, use of a small number of microsatellite markers lowers the accuracy of the results. Thus, a suitable number of microsatellite markers is necessary to establish a genetic monitoring system. In this study, we screened all 137 known microsatellite loci in the gerbil and selected 39 highly polymorphic markers that are uniformly distributed on mouse chromosomes. As detailed above, we tested these markers in gerbil populations and found that 28 of the 39 microsatellite markers could be used to establish an effective microsatellite marker system. This conclusion was based on the results of independent sample $t$-tests of the different microsatellite markers with regard to mean effective allele number, mean of Shannon's information index, and mean $H_{\mathrm{E}}$ (Tables S1, $\underline{\mathbf{S 2}}$, and $\underline{\mathbf{S 3}}$ ). Barker (1994) suggested that at least 25 randomly selected animals should be used in population and diversity studies and that loci with at least four different alleles should be used to reduce the standard errors of estimated distances. Thus, the 28 gerbil microsatellite markers selected here are appropriate for genetic monitoring and breeding. Future developments in gerbil genetic research will 
undoubtedly result in the identification of more microsatellite markers and in determination of the chromosomal distributions. Such new information would allow the monitoring system to be adjusted and improved. To date, however, our system is the most best available for genetic quality control in gerbils.

We used the selected 28 microsatellite markers to evaluate genetic variation in five gerbil populations in China. Most of the 28 microsatellite markers departed significantly from the HWE. This result was supported by differences between $H_{\mathrm{E}}$ and $H_{\mathrm{O}}$, indicating the existence of non-random mating. Overall, $H_{\mathrm{E}}, N_{\mathrm{A}}$ and I values were greatest in the NW population and lowest in the GZ population (Table 2). The NW population was derived from 31 gerbils captured from the wild (Figure 1). This may explain why $H_{\mathrm{E}}, N_{\mathrm{A}}$ and I values were the greatest in this population. It is notable that these indicators of genetic variation in NW were similar to those in the BJ population (Table 2). There are two possible reasons for this similarity: first, the BJ population was derived from approximately 400 pairs of gerbils captured in 1986 from the wild in Hohehot Municipality, China, and have been maintained in the laboratory for 23 years; second, the NW samples might share some degree of genetic relationship. The GZ population was established from 40 pairs of gerbils from the ZJ, BJ, and DL populations, and it was maintained as a relatively small population. These maintenance procedures might have produced the relatively low $H_{\mathrm{E}}, N_{\mathrm{A}}$, I, and $H_{\mathrm{O}}$ values (Table 2). The $H_{\mathrm{O}}$ values were lower than $H_{\mathrm{E}}$ values in four populations, and only the GZ population had an $H_{\mathrm{O}}$ value higher than the $H_{\mathrm{E}}$ value (Table 2). This suggests that either random mating has not occurred in the GZ population or that there are additional unknown subdivisions that have not been identified.

The pairwise $R_{\mathrm{ST}}, D_{\mathrm{S}}$ and neighbour-joining results suggested relatively large differentiation of GZ and other gerbil populations. The marked differences between the populations could be explained by the differences in their origins and mating systems. Although the GZ population was partly derived from the DL population, these two populations nevertheless showed significant genetic differentiation (Table 3). Possibly, the length of time since separation of the populations, combined with the introduction of $\mathrm{HZ}$ and BJ population characteristics, and the influence of human selection might have contributed to the high $R_{\mathrm{ST}}$ and $D_{\mathrm{S}}$ values (Table 3). The BJ and NW populations were genetically close because of their similar origins, having been captured in the same area. AMOVA revealed that variance among individuals within populations accounted for $69.82 \%$ of total variance, while variance among populations accounted for $29.62 \%$ (Table 4 ), indicating that the microsatellite marker system developed here could identify the genetic variation of the five populations.

The polymorphic and microsatellite variations in the BJ laboratory population showed no significant differences to those of wild gerbils. Du et al. (2010) similarly reported $H_{\mathrm{O}}>$ $H_{\mathrm{E}}$ using 12 microsatellite DNA markers. Ding et al. (2008) also reported an absence of significant genetic variation between a laboratory gerbil population (Z: ZCLA) and wild gerbils (captured from Shan Xi Province, China) using microsatellite DNA markers. However, Neumann et al. (2001) found a lower level of genetic variation in laboratory than wild gerbils. Low genetic variability was also identified in laboratory gerbils by Razzoli et al. (2003). These different conclusions may be the result of the differences in the breeding history of the animals studied and of the different microsatellite markers used to screen for genetic variation. The optimized microsatellite marker system used in our research was based on a statistical method and involved screening relatively large numbers of microsatellite markers and DNA samples. In addition, the $H_{\mathrm{E}}$ of the $\mathrm{BJ}$ and $\mathrm{NW}$ populations in this study was higher than in the $\mathrm{CMU}, \mathrm{HOH}$, and YIN populations. The conflicting outcomes between Du et al. (2010) 
and here might have been caused by the use of different populations and loci. The CMU sample used by Du et al. (2010) was from same population as the BJ sample here. However, the HOH and YIN samples in the Du et al. (2010) report were collected from a smaller area than the NW sample here; this may account for the higher $H_{E}$ of the BJ and NW populations in our study to that of the $\mathrm{CMU}, \mathrm{HOH}$, and YIN populations. The BJ colony was established from approximately 400 pairs of wild gerbils captured in 1986 from the Hohehot Municipality, China. In contrast, almost all of the laboratory gerbils used in United States and European laboratories have been derived from a source that was established from 20 pairs of wild gerbils captured from Manchuria (Inner mongolia, China) in 1935. The strain of laboratory gerbils used by Neumann et al. (2001) is also from this resource. To maintain genetic variation, the BJ population has been outbred; this will undoubtedly have contributed to its higher level of genetic variability. Our results demonstrated that laboratory and wild gerbils did not show differences in genetic variation. However, Neumann et al. (2001) reported that allele numbers were higher in wild gerbils than laboratory gerbils. This discrepancy might be related to the different sources of the wild animals. The wild gerbils in this study were captured from an area at the junction of the Inner Mongolia Autonomous Region and Ningxia Province, China. The wild gerbils used by Neumann et al. (2001) were "trapped at six different locations about 130 to $140 \mathrm{~km}$ southwest and $100 \mathrm{~km}$ west of Ulaanbaatar".

In this study, we have developed a microsatellite marker system for the genetic monitoring of laboratory populations of gerbils. This system will be helpful and important for the genetic quality control and monitoring of these populations. The level of heterozygosity in four laboratory gerbil populations was lower than expected emphasizing that standard operation principles, strict supervision and the microsatellite marker system presented here should be implemented for the maintenance of gerbil populations in China to obtain genetically defined gerbils for use in biomedical research. The identification here of genetic differences among the four laboratory gerbil populations emphasizes the need to consider the possible effects of genetic variation on the outcome of experiments depending on the population used.

\section{Conflicts of interest}

The authors declare no conflict of interest.

\section{ACKNOWLEDGMENTS}

Research supported by the National Science Foundation of China (\#31172168 and \#31272393), and the Key Projects in the National Science \& Technology Pillar Program (\#2015BAI09B01).

\section{Supplementary material}

\section{REFERENCES}

Barker JSF (1994). A global protocol for determining genetic distance among domestic livestock breeds. Proceedings of the 5th World Congress on Genetics Applied to Livestock Production 21: 501-508.

Beck J, Stummer W, Lehmberg J, Baethmann A, et al. (2007). Activation of leukocyte-endothelial interactions and reduction of selective neuronal death after global cerebral ischemia. Neurosci. Lett. 414: 159-164.

Beckmann JS and Soller M (1990). Toward a unified approach to genetic-mapping of eukaryotes based on sequence 
tagged microsatellite sites. Biotechnology 8: 930-932.

Cheal M (1986). The gerbil: a unique model for research on aging. Exp. Aging Res. 12: 3-21.

Chiba T, Marusawa H, Seno H and Watanabe N (2008). Mechanism for gastric cancer development by Helicobacter pylori infection. J. Gastroenterol. Hepatol. 23: 1175-1181.

Conchedda M, Gabriele F and Bortoletti G (2006). Development and sexual maturation of Echinococcus granulosus adult worms in the alternative definitive host, Mongolian gerbil (Meriones unguiculatus). Acta Trop. 97: 119-125.

Cordeiro RS, Scarano WR, Campos SGP, Santos FCA, et al. (2008). Androgen receptor in the Mongolian gerbil ventral prostate: evaluation during different phases of postnatal development and following androgen blockage. Micron 39: $1312-1324$.

Deming DM, Boileau AC, Lee CM and Erdman JW (2000). Amount of dietary fat and type of soluble fiber independently modulate postabsorptive conversion of beta-carotene to vitamin A in mongolian gerbils. J. Nutr. 130: 2789-2796.

Dietrich WF, Miller J, Steen R, Merchant MA, et al. (1996). A comprehensive genetic map of the mouse genome. Nature 380: 149-152.

Ding XM, Qian BZ, Matsuda J, Koura M, et al. (2008). Genetic diversity of Mongolian gerbils (Meriones unguiculatus). Yi Chuan 30: 877-884.

Dowden J and Corbett D (1999). Ischemic preconditioning in 18- to 20-month-old gerbils: long-term survival with functional outcome measures. Stroke 30: 1240-1246.

Du XY, Chen ZW, Li W, Tan YQ, et al. (2010). Development of novel microsatellite DNA markers by cross-amplification and analysis of genetic variation in gerbils. J. Hered. 101: 710-716.

Du XY, Zhu XD, Chen ZW, Lu J, et al. (2011). Characteristics of circle of Willis variations in the mongolian gerbil and a newly established ischemia-prone gerbil group. ILAR 52: e1-e7.

Excoffier L, Laval G and Schneider S (2005). Arlequin (version 3.0): an integrated software package for population genetics data analysis. Evol. Bioinform. Online 1: 47-50.

Fujisawa N, Maeda Y, Yamamoto Y, Sato NL, et al. (2003). Newly established low seizure susceptible and seizure-prone inbred strains of Mongolian gerbil. Exp. Anim. 52: 169-172.

Goodfellow PN (1993). Viewpoint: microsatellites and the new genetic maps. Curr. Biol. 3: 149-151.

Hirayama F, Takagi S, Kusuhara H, Iwao E, et al. (1996). Induction of gastric ulcer and intestinal metaplasia in Mongolian gerbils infected with Helicobacter pylori. J. Gastroenterol. 31: 755-757.

Hossmann KA (1998). Experimental models for the investigation of brain ischemia. Cardiovas. Res. 39: 106-120.

Kato N, Nonaka N, Oku Y and Kamiya M (2005). Immune responses to oral infection with Echinococcus multilocularis protoscoleces in gerbils: modified lymphocyte responses due to the parasite antigen. Parasitol. Res. 96: 12-17.

Kirino T and Sano K (1984). Selective vulnerability in the gerbil hippocampus following transient ischemia. Acta Neuropathol. 62: 201-208.

Levine S and Sohn D (1969). Cerebral ischemia in infant and adult gerbils. Relation to incomplete circle of Willis. Arch. Pathol. 87: 315-317.

Love JM, Knight AM, McAleer MA and Todd JA (1990). Towards construction of a high-resolution map of the mouse genome using PCR-analyzed microsatellites. Nucleic Acids Res. 18: 4123-4130.

Marklund S, Ellegren H, Eriksson S, Sandberg K, et al. (1994). Parentage testing and linkage analysis in the horse using a set of highly polymorphic microsatellites. Anim. Genet. 25: 19-23.

Mogil JS (1999). The genetic mediation of individual differences in sensitivity to pain and its inhibition. Proc. Natl. Acad. Sci. U. S. A. 96: 7744-7757.

Nei M (1972). Genetic distances between populations. Am. Nat. 106: 283-292.

Nei M (1987). Molecular evolutionary genetics. Columbia University Press, New York.

Neumann K, Maak S, Stuermer IW, von Lengerken G, et al. (2001). Low microsatellite variation in laboratory gerbils. $J$. Hered. 92: 71-74.

Paetkau D, Calvert W, Stirling I and Strobeck C (1995). Microsatellite analysis of population structure in Canadian polar bears. Mol. Ecol. 4: 347-354.

Paetkau D, Waits LP, Clarkson PL, Craighead L, et al. (1997). An empirical evaluation of genetic distance statistics using microsatellite data from bear (Ursidae) populations. Genetics 147: 1943-1957.

Page RDM (1996). TreeView: an application to display phylogenetic trees on personal computers. Comput. Appl. Biosci. 12: 357-358.

Pereira S, Martins OA, Domeniconi RF, Pinheiro PFF, et al. (2009). Vasectomy increase cell proliferating in gerbil prostate on cancer chemical induction model. J. Androl. 30: 97-98.

Raymond M and Rousset F (1995). GENEPOP (Version 1.2): population genetics software for exact tests and ecumenicism. J. Hered. 86: 248-249.

Razzoli M, Papa R, Valsecchi P and Nonnis Marzano F (2003). AFLP to assess genetic variation in laboratory gerbil 
(Meriones unguiculatus). J. Hered. 94: 507-511.

Shibata W, Hirata Y, Maeda S, Ogura K, et al. (2006). CagA protein secreted by the intact type IV secretion system leads to gastric epithelial inflammation in the Mongolian gerbil model. J. Pathol. 210: 306-314.

Song G (1999). Epidemiological progresses of hemorrhagic fever with renal syndrome in China. Chin. Med. J. 112: 472477.

Vyse TJ and Todd JA (1996). Genetic analysis of autoimmune disease. Cell 85: 311-318.

Waser PM and Strobeck C (1998). Genetic signatures of interpopulation dispersal. Trends Ecol. Evol. 13: 43-44.

Watanabe M, Lee BJ, Kamitani W, Kobayashi T, et al. (2001). Neurological diseases and viral dynamics in the brains of neonatally borna disease virus-infected gerbils. Virology 282: 65-76.

Weber JL (1990). Informativeness of human (dC-dA) $)_{n} \cdot(d G-d T)_{n}$ polymorphisms. Genomics 7: 524-530. 